Mit einem solchen Denken, das zum Beispiel in den Verhandlungen um die Neuordnung der Finanzierung zum Ausdruck kommt, könne man die Forderungen nicht bestehen, welche die Zukunft an die wissenschaftliche und technologische Entwicklung der Bundesrepublik stellen werde. Gleichwohl kann die MPG mit der bisherigen finanziellen Entwicklung relativ zufrieden sein, und kein Bundesland ist jetzt mehr von Aktivitäten der MPG ausgenommen, nachdem nunmehr auch das Saarland und Bremen ein Institut erhalten.

Größeren Kummer bereitet die Entwicklung in der Europäischen Gemeinschaft. Der Abstand zwischen den Nord- und Südländern ist im Bereich der Wissenschaft sehr groß. Der Brüsseler Kommission trauen die Wissenschaftler nicht zu, daß sie das von ihr selbst angestrebte „Europa der Forscher“ zuwege bringen werde. Dieses müsse vielmehr von der Basis her wachsen. Die MPG hat daher, zusammen mit der DFG und anderen Wissenschaftsorganisationen, eine Initiative ergriffen.

Als einen außerordentlich wirksamen Katalysator für intensive Kontakte und Kooperationen pries Staab in Wiesbaden "Europäische Forschungs-Konferenzen“, die nach dem Vorbild der amerikanischen Gordon-Konferenzen eingerichtet werden sollen. In relativ eng umschriebenen Forschungsgebieten bringen sie führende Vertreter des jeweiligen Faches mit jungen Nachwuchswissenschaftlern in einem informellen Rahmen und relativ kleinen Kreis von jeweils etwa hundert Personen zusammen.

Ein europäisches Austauschprogramm soll die Konferenzen ergänzen. Hervorragende Postdoktoranden sollen in diesem Rahmen für einige Zeit an ein exzellentes Institut in einem anderen europäischen Land gehen. Allerdings müsse sichergestellt werden, daß sie auch mindestens für einige Jahre in ihr Heimatland zurückkehren. Beide Programme sollen aus Mitteln der EG-Kommission über die Europäische Wissenschafts-Stiftung finanziert werden.

Außerdem hat das Bundesforschungsministerium der MPG einen Preis vorgeschlagen, der die Kooperation eines deutschen und eines ausländischen Forschers und ihrer Arbeitsgruppen vorsieht.

\section{Medizin-Studium soll international aufgewertet und interdisziplinär werden}

Ein neues, dem internationalen Standard entsprechendes Curriculum für das Medizinstudium soll eine interdisziplinäre Arbeitsgruppe des Wissenschaftsrates vorbereiten. Die im Dezember 1988 verabschiedeten „Empfehlungen zur Verbesserung der Ausbildungsqualität in der Medizin“ wurden, obwohl als Sofortmaßnahme gedacht, noch immer nicht umgesetzt. Sie sehen vor, die Anfängerzahlen für die Humanmedizin nach der patientenbezogenen Aufnahmekapazität festzusetzen, die Zulassungszahlen für den gesamten Studiengang Medizin einheitlich festzulegen und die Gruppengrößen für Studierende zu vermindern. Klagen aus dem Ausland über die vielfältigen Lücken in der deutschen Medizinerausbildung werden sehr ernst genommen. Die Arbeitsgruppe soll mittelfristig darauf hinarbeiten, daß das Studium verkürzt wird, wobei gleichzeitig wissenschaftliche und praktische Anforderungen berücksichtigt werden, daß die Trennung zwischen vorklinischer und klinischer Ausbildung überwunden wird und daß Umfang und Stellung der naturwissenschaftlichen Grundlagenfächer festgelegt werden.

Die Zahl der Studienplätze in der Medizin soll nach den begonnenen Ausbauvorhaben rund 65000 betragen. Insgesamt sind 811000 Plätze vorgesehen. Im vorigen Studienjahr waren 1,4 Millionen Studenten eingeschrieben, 11000 der 225000 Studienanfänger waren Mediziner.

Der Arbeitsgruppe gehören folgende Professoren als Mitglieder des Wissenschaftsrates an: Jürgen van de Loo (Internist, Münster; Vorsitzender), Horst Franz Kern (Zellbiologe und -pathologe, Marburg), Gerhard Neuweiler (Zoologe, München), Peter C. Scriba (Internist, Lübeck), Widmar Tanner (Botaniker, Regensburg). Sie werden um weitere Sachverständige sowie Vertreter der Bundes- und Länderministerien ergänzt.

\section{Personen und Institutionen}

Der Jurist Dr. Winfried Benz ist neuer Generalsekretär des Wissenschaftsrates. Seit 1976 war er Kanzler der Universität Mannheim. Sein Vorgänger Dr. Peter Kreyenberg ist seit 31. Mai 1988 Staatssekretär bei der schleswig-holsteinischen Ministerin für Bildung, Wissenschaft, Jugend und Kultur. Als Nachfolger von Prof. Dr. Gotthilf Hempel hat der Vorsitzende des Stiftungsvorstands des Deutschen Krebsforschungszentrums Heidelberg, Prof. Dr. Harald zur Hausen, am 1. Juli sein Amt als Vorsitzender der Arbeitsgemeinschaft der Großforschungseinrichtungen (AGF) angetreten. Er wurde für zunächst zwei Jahre gewählt. - Am 22. September wird Prof. Dr. Reimar Lüst die Geschäfte des Präsidenten der Alexander von Humboldt-Stiftung übernehmen. Bis ins Jahr 1990 bleibt er gleichzeitig Generaldirektor der Europäischen Weltraumorganisation ESA in Paris. Sein Vorgänger, der Bonner Physiker Prof. Dr. Wolfgang Paul, bleibt Ehrenpräsident der AvH. - Prof. Dr. Wolfgang Wild, früherer bayerischer Kultusminister und vorher Präsident der Technischen Universität München, ist jetzt Generaldirektor der neuen Deutschen Agentur für Raumfahrtangelegenheiten (DARA) in Bonn. Administrativer Geschäftsführer der DARA ist Dr. Wolfgang Grillo, seit einem Jahr Kanzler der Universität München und vorher kaufmännischer Geschäftsführer des Instituts Max von Laue/Paul Langevin (ILL) in Grenoble, danach der Gesellschaft für Strahlen- und Umweltforschung in München.

\section{Erfolge und Probleme der neurogenetischen Forschung}

Die molekulare Neurogenetik sieht Dr. Wolfgang Wille von der Arbeitsgruppe für Molekulare Neurogenetik am Institut für Genetik der Universität Köln einer Diffamierungskampagne ausgesetzt: Sie könne nicht, wie ihr vorgeworfen werde, auf absehbare Zeit erklären, wie wir denken und fühlen, wie wir Gedanken und Information speichern und wieder 